\title{
Characterization and expression analysis of circadian clock genes in the diploid woodland strawberry Fragaria vesca
}

\author{
X.D. CHEN, J. WANG, M.Z. ZHAO*, and F. ZHAO \\ Institute of Pomology, Jiangsu Academy of Agricultural Sciences and Jiangsu Key Laboratory for Horticultural \\ Crop Genetic Improvement, Nanjing 210014, P.R. China
}

\begin{abstract}
Strawberry is an economically important fruit crop worldwide. Circadian clock genes are endogenous timers that regulate a wide range of metabolic processes and consequently plant development. However, little is known about the circadian clock genes in strawberry. In the present work, we identified 12 primary circadian clock genes from the diploid woodland strawberry (Fragaria vesca L.) genome. Phylogenetic, conserved motif, and gene structure analyses revealed the evolutionary relationships of strawberry circadian clock genes with homologous genes from other species. Promoter analysis revealed different regulatory elements responding to abiotic and biotic stresses and phytohormones. We characterized the transcript patterns of strawberry circadian clock genes over a 48-h period. The expression patterns of seven circadian clock genes displayed circadian rhythms. We also examined the expression patterns of these genes in response to low-temperature stress and six of them showed an upregulated expression. Interestingly, most of these upregulated genes were highly expressed during the day. Our study reveals the characteristics of primary circadian clock components in diploid woodland strawberry and their responses to low-temperature stress and lays a foundation for future functional studies of these circadian clock genes during the growth and development of diploid woodland strawberry.
\end{abstract}

Additional key words: Arabidopsis thaliana, cold stress, gene expression, phylogenetic analysis.

\section{Introduction}

Circadian rhythms are found in a wide variety of organisms ranging from cyanobacteria to mammals (Dong and Golden 2008, Wang et al. 2017). The periodicity of circadian rhythms is approximately $24 \mathrm{~h}$, which is consistent with the light-dark cycle present on Earth (Greenham and McClung 2015). Circadian rhythms are driven by an endogenous circadian clock (Matsushika et al. 2000). The circadian clock of Arabidopsis is composed of multiple interlocking transcriptiontranslation feedback loops (Dong et al. 2011, Maibam et al. 2013, Hsu and Harmer 2014). Different circadian clock proteins function at certain times during the day and night to maintain circadian rhythms (Hsu and Harmer 2014). Two morning-phased components circadian clockassociated 1 (CCA1) and late elongated hypocotyl (LHY), along with one evening-phased component timing of

CAB 1 (TOC 1), comprise the core circadian feedback loop in Arabidopsis (Dong et al. 2011). CCA1 and LHY are MYB transcription factors that have partially redundant functions (Hsu and Harmer 2014). The expression of $C C A 1$ and $L H Y$ peaks at dawn, whereas the expression of TOCl is greatest in the early evening (Dong et al. 2011). TOC1 (also known as pseudoresponse regulator 1 (PRR1)), together with PRR9, PRR7, PRR5, and PRR3, plays a key role in the circadian clock (Matsushika et al. 2000). The expression of TOC1 is repressed by $C C A 1$ and $L H Y$, while $T O C 1$ is required for the induction of CCAI and $L H Y$ (Alabadi et al. 2002, Dong et al. 2011). PRR9, PRR7, and PRR5 play redundant roles in repressing the expression of $C C A 1$ and LHY (Nakamichi et al. 2010). CCA1 and LHY belong to the small REVEILLE subfamily, in which several

Submitted 7 August 2017, last revision 22 November 2017, accepted 11 December 2017.

Abbreviations: BOA - brother of lux arrhythmo; CCA1 - circadian clock-associated 1; CHE - CCA1 hiking expedition; ELF - early flowering; LHY - late elongated hypocotyl; LUX - lux arrhythmo; PRR - pseudo-response regulator; qPCR - quantitative polymerase chain reaction; RVE - REVEILLE; TOC 1 - timing of CAB 1; ZTL - ZEITLUPE.

Acknowledgements: This work was supported by the Jiangsu Planned Projects for Postdoctoral Research Funds (No. 1601103B), the Jiangsu Provincial Natural Science Foundation of China (No. BK20130722), and the National Natural Science Foundation of China (No. 31301764).

* Corresponding author; e-mail: njzhaomz@163.com 
homologues of CCA1/LHY, i.e., REVEILLE 8 (RVE8), $R V E 4$, and $R V E 6$ play important roles in regulating the expression of many evening-phase genes (Rawat et al. 2011, Hsu and Harmer 2014). In the circadian clock system, evening-phase components include PRR5, TOC1, CHE (CCA1 hiking expedition), LUX (LUX arrhythmo), $B O A$ (brother of lux arrhythmo), ELF3 (early flowering 3), ELF4, and ZTL (ZEITLUPE) (Hsu and Harmer 2014). $C H E$ has a function in repressing the expression of $C C A 1$ (Pruneda-Paz et al. 2009). LUX, ELF3, and ELF4 form the "evening complex", which negatively regulates the expression of PRR9 (Nusinow et al. 2011, Herrero et al. 2012, Hsu and Harmer 2014). ZTL is an F-box protein that targets TOC1 and PRR5 for ubiquitination (Mas et al. 2003, Hsu and Harmer 2014).

Many circadian clock components take part in regulating different biological processes, such as the diurnal control of hypocotyl elongation, photosynthesis, and saccharide metabolism, as well as abiotic and biotic stress responses (Hsu and Harmer 2014, Greenham and McClung 2015). The CCA1 and evening complex proteins (ELF3, ELF4, and LUX) are involved in regulating hypocotyl elongation (Nusinow et al. 2011, Campoli et al. 2012). In addition, CCA1 and LHY have been reported to regulate carbon partitioning towards starch (Greenham and McClung 2015). In Arabidopsis, PRR9, PRR7, and PRR5 negatively regulate the biosynthetic pathways of chlorophyll, carotenoids, abscisic acid, and $\alpha$-tocopherol (Fukushima et al. 2009). Several components of the circadian clock system (CCA1, LHY, PRR9, 7, and 5) have been implicated in regulating low-temperature stress (Fukushima et al. 2009, Dong et al. 2011, Hsu and Harmer 2014).

The circadian clock has been well studied in Arabidopsis, and its importance in controlling key agricultural traits is becoming clear (Hsu and Harmer 2014, Bendix et al. 2015). A number of circadian clock system proteins have been identified and analysed in many crops, such as Castanea sativa (Ibanez et al. 2008), Oryza sativa (Yang et al. 2013), Brassica rapa (Lou et al. 2012), and Glycine max (Marcolino-Gomes et al. 2014). Until recently, circadian clock system genes have not been well characterized in the strawberry genome. To date, only a few circadian clock components and their potential roles in regulating flowering time in strawberry have been reported (Mouhu et al. 2009).

The cultivated strawberry (Fragaria $\times$ ananassa Duch.) is an important horticultural crop throughout the world (Wei et al. 2016). F. $\times$ ananassa is an octoploid strawberry, harbouring eight sets of chromosomes $(2 \mathrm{n}=$ $8 \mathrm{x}=56$ ), which complicates genetic studies (Shulaev et al. 2011). By comparison, the diploid woodland strawberry (Fragaria vesca) has a small and sequenced genome $(2 \mathrm{n}=14,240 \mathrm{Mb})$ that is highly congenic with octoploid cultivated strawberry (Shulaev et al. 2011), making it a good platform to study circadian clock genes in strawberry. The aim of the present work was to isolate and characterize 12 primary circadian clock genes from $F$. vesca and to conduct a broad bioinformatics analysis, including chromosome position, evolutionary relationships, protein motifs, gene structure, and cis-acting elements. We further investigated the expression profiles of these genes at different times as well as in response to cold stress.

\section{Materials and methods}

Plants, growth conditions and treatments: A seventhgeneration inbred line Rugen F7-4 of Fragaria vesca L. (gift from Dr. Gu; for more information about this lane see $\mathrm{Gu}$ et al. 2016) was used in this study. Strawberry seedlings were grown at a temperature of $24^{\circ} \mathrm{C}$, a relative humidity of $60 \%$, a $10-\mathrm{h}$ photoperiod, and an irradiance of $200 \mu \mathrm{mol} \mathrm{m} \mathrm{m}^{-2} \mathrm{~s}^{-1}$. Fully expanded leaves from 8-weekold seedlings were collected for real-time qPCR analysis at 4-h intervals from Zeitgeber time 0 (ZT0) to ZT48. For cold treatments, strawberry seedlings were transferred to $4{ }^{\circ} \mathrm{C}$ for $24 \mathrm{~h}$. Control plants were maintained at $24^{\circ} \mathrm{C}$. In addition, leaves were collected at 4 - $\mathrm{h}$ intervals starting at ZT0 for $48 \mathrm{~h}$. All samples were immediately put into liquid $\mathrm{N}_{2}$ and stored at $-80^{\circ} \mathrm{C}$ before RNA extraction.

Identification of strawberry circadian clock genes: To identify strawberry circadian clock genes, we downloaded the amino acid sequences of Arabidopsis circadian clock proteins from TAIR (http:// www.arabidopsis.org/; Lamesch et al. 2012) and used them as query sequences to conduct BLASTP searches in the $F$. vesca genome (fvesca v1.0 genemark hybrid) database at the genome database for Rosaceae (GDR,

http:/www.rosaceae.org; Jung et al. 2014). The top hit sequences were selected on the basis of whether they had an E-value close to 0, using a cutoff E-value of 1e-30. The circadian clock protein sequences from peach (Prunus persica v.2.1), tomato (Solanum lycopersicum iTAG2.4), rice (Oryza sativa v7 JGI), and maize (Zea mays Ensembl-18) were obtained from Phytozome (https://phytozome.jgi.doe.gov/pz/portal.html) using the same BLASTP method (details listed in Table 1 Suppl.). The identified strawberry circadian clock genes were mapped to chromosomes using MapInspect software (http://www.files32.com/MapInspect-i366074.asp).

Phylogenetic analysis and gene structure: Phylogenetic trees of circadian clock proteins were generated using Molecular evolutionary genetic analysis (MEGA) 5.0 software (Tamura et al. 2011). The neighbour-joining method was applied to construct circadian clock trees with the p-distance model, pairwise deletion of gaps, and bootstrapping (1000 replicates; random seed). Conserved protein motif analysis was performed by online Multiple expectation-maximization for motif elicitation (MEME) v. 4.10.2; http://meme-suite.org/tools/meme) (Bailey et al. 
2009). The parameters were as follows: minimum length of the motif, 6; maximum length of the motif, 100; and maximum number of motifs, 10 . The genomic and coding sequences of strawberry circadian clock genes were retrieved from GDR. Then, these genomic and coding sequences were analysed using the online program gene structure display server (GSDS) 2.0 ( $\mathrm{Hu}$ et al. 2015) to infer the exon-intron distribution of strawberry circadian clock genes. The whole genomic sequences of strawberry circadian clock genes were retrieved from National Center for Biotechnology Information (NCBI, http://www. ncbi.nlm.nih.gov/). The $1.5 \mathrm{~kb}$ upstream regions of the translation start sites of the strawberry circadian clock genes were used for regulatory elements analysis using PlantCARE (http://bioinformatics.psb. ugent.be/webtools/ plantcare/html/) (Lescot et al. 2002).

RNA extraction and gene expression analysis: Total RNA was extracted from frozen leaves using the $P D$ kit (Pudishengwu, Shanghai, China). The gDNA ERASER in the kit was used to remove gDNA from RNA samples. Then, RNAs were reverse-transcribed into cDNA using
BioTeke (Beijing, China) SupermoIII RT kit. Each realtime qPCR was performed with a total volume of $20 \mathrm{~mm}^{3}$ consisting of $10 \mathrm{~mm}^{3}$ of SYBR Green Premix Ex Taq (TaKaRa, Tokyo, Japan), $0.8 \mathrm{~mm}^{3}$ of $1.0 \mu \mathrm{M}$ of each primer, and $7.4 \mathrm{~mm}^{3}$ of sterile distilled $\mathrm{H}_{2} \mathrm{O}$ using the 7500 real-time PCR system (Applied Biosystems, New York, USA). The amplification conditions were as follows: $95^{\circ} \mathrm{C}$ for $10 \mathrm{~min}$; 39 cycles of $95^{\circ} \mathrm{C}$ for $15 \mathrm{~s}$ and $60{ }^{\circ} \mathrm{C}$ for $40 \mathrm{~s}$; then, the temperature was gradually increased by $0.5^{\circ} \mathrm{C}$ every $10 \mathrm{~s}$ to perform a melting curve analysis. The real-time $\mathrm{qPCR}$ results are presented as $2^{-\Delta \mathrm{CT}}$ values (Schmittgen and Livak 2008), which were normalized to GAPDH (Gu et al. 2016) using the $\triangle \mathrm{CT}$ method (Schmittgen and Livak 2008). The expression data were analysed using Microsoft Excel 2010. Student's $t$-test was applied to compare statistical significances between the low-temperature treated and the control samples. The primers used in this study are listed in Table 2 Suppl. The real-time qPCR results were confirmed by three biological repetitions. Each biological repetition was performed with three technical repetitions.

\section{Results}

A total of 16 Arabidopsis primary circadian clock genes CCA1, LHY, PRR9, PRR7, RVE8, RVE4, RVE6, PRR5, PRR3, TOC1, CHE, LUX, BOA, ELF3, ELF4, and ZTL (Hsu and Harmer 2014), were used for a BLASTP search in $G D R$ to identify primary circadian clock genes in the $F$. vesca genome (fvesca_v1.0_genemark_hybrid). As a result, we identified $1 \overline{2}$ circadian clock genes in strawberry (Table 1). Detailed information (including gene location, genomic length, and number of amino acids) regarding each gene is listed in Table 1. These

genes were distributed over six chromosomes. Four circadian clock genes were mapped to chromosome 7 , and two circadian clock genes were found on chromosome 6. Chromosomes 2, 3, and 4 contained one circadian clock gene each (Fig. 1).

CCA1/LHY and TOC1 (PRRI) comprise the core circadian feedback loop (Dong et al. 2011). To gain a better understanding of the potential function and evolutionary relationships of strawberry CCA1/LHY and TOC1 (PRR1), we constructed phylogenetic trees of

Table 1. Characteristics of primary circadian clock genes in Arabidopsis and Fragaria vesca. ${ }^{\text {a }}$ - Hsu and Harmer (2014); ${ }^{\text {b }}$ IDs, gene length, and the number of amino acids (Fragaria vesca whole genome v. 1.0. build 8 assembly and annotation) are available at genome database for Rosaceae (http:/www.rosaceae.org).

\begin{tabular}{|c|c|c|c|c|c|c|c|}
\hline $\begin{array}{l}\text { AGI } \\
\text { (Arabidopsis) }\end{array}$ & $\begin{array}{l}\text { Name } \\
\text { (Arabidopsis) }\end{array}$ & 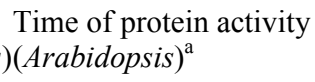 & $\begin{array}{l}\text { Gene Id } \\
(F . \text { vesca })^{\mathrm{b}}\end{array}$ & $\begin{array}{l}\text { Name } \\
(F . \text { vesca })\end{array}$ & Gene location & $\begin{array}{l}\text { Length } \\
{[\mathrm{bp}]^{\mathrm{b}}}\end{array}$ & $\begin{array}{l}\text { Number } \\
\text { of } \mathrm{aa}^{\mathrm{b}}\end{array}$ \\
\hline AT2G46830 & $A t C C A 1$ & morning & -- & & & & \\
\hline AT1G01060 & AtLHY & morning & gene 18601 & $F v L H Y$ & LG7:2710468 to 2713025 & 2558 & 712 \\
\hline AT2G46790 & AtPRR9 & after dawn & gene 18611 & $F v P R R 9$ & LG7:2760853 to 2764189 & 3337 & 658 \\
\hline AT5G02810 & AtPRR7 & after dawn & gene 18151 & $F v P R R 7$ & LG6:10027241 to 10031868 & 4628 & 765 \\
\hline AT3G09600 & AtRVE8 & afternoon & gene18104 & $F v R V E 8$ & LG6:10090024 to 10092841 & 2818 & 276 \\
\hline AT5G02840 & AtRVE4 & presumably afternoon & -- & & & & \\
\hline AT5G52660 & AtRVE6 & presumably afternoon & gene 13188 & $F v R E V 6$ & LG7:22153843 to 22157234 & 3392 & 348 \\
\hline AT5G24470 & AtPRR5 & afternoon & gene 12454 & $F v P R R 5$ & LG7:20753894 to 20757515 & 3622 & 672 \\
\hline AT5G60100 & AtPRR3 & evening & -- & & & & \\
\hline AT5G61380 & AtTOC1 & evening & gene 26055 & FvTOC1 & LG5:8058964 to 8063051 & 4088 & 536 \\
\hline AT5G08330 & AtCHE & evening & gene 12294 & $F v C H E$ & LG5:13952649 to 13953503 & 855 & 284 \\
\hline AT3G46640 & AtLUX & evening & gene 30204 & $F v L U X$ & LG3:19000151 to 19001107 & 957 & 318 \\
\hline AT5G59570 & $A t B O A$ & evening & -- & & & & \\
\hline AT2G25930 & AtELF3 & evening & gene 02656 & $F v E L F 3$ & LG2:20296037 to 20299458 & 3422 & 769 \\
\hline AT2G40080 & AtELF4 & evening & gene 20968 & $F v E L F 4$ & LG7:18198444 to 18201502 & 3059 & 304 \\
\hline AT5G57360 & AtZTL & evening & gene16999 & $F v Z T L$ & LG4:3187275 to 3188789 & 1515 & 504 \\
\hline
\end{tabular}


CCA1/LHY and PRR family genes by comparing their protein sequences in Arabidopsis, peach, tomato, maize, rice, and strawberry. Meanwhile, the conserved motifs and exon/intron organization of these proteins were analysed to obtain further insight into the relationships between these orthologues. The phylogenetic results showed that FvLHY and PpLHY had a close evolutionary relationship. The protein motif analysis showed that motifs 2, 3, 4, 5, 6, 9, and 10 were found in all 9 CCA1/LHY proteins. Motifs 8 and 9 were identified in OsCCA1 and ZmCCA1/ZmLHY (Fig. 2). The exon/intron distribution results showed that $F v L H Y$ and $Z m L H Y$ contained three phase 0 introns, while the other CCA1/LHY genes usually had 5 - 6 introns (Fig. 1 Suppl.). As illustrated by Fig. 3, strawberry PRR proteins had a close evolutionary relationship with those of peach. Motif 2 and motif 4 were observed in all PRR proteins. In addition, the majority of PRR proteins contained motif 1 and motif 3, except for SIPRR3 and ZmPRR5. These conserved motifs may contribute to the common functions of PRR family proteins. The gene structure analysis of $P R R$ family genes is shown in Fig. 2 Suppl.

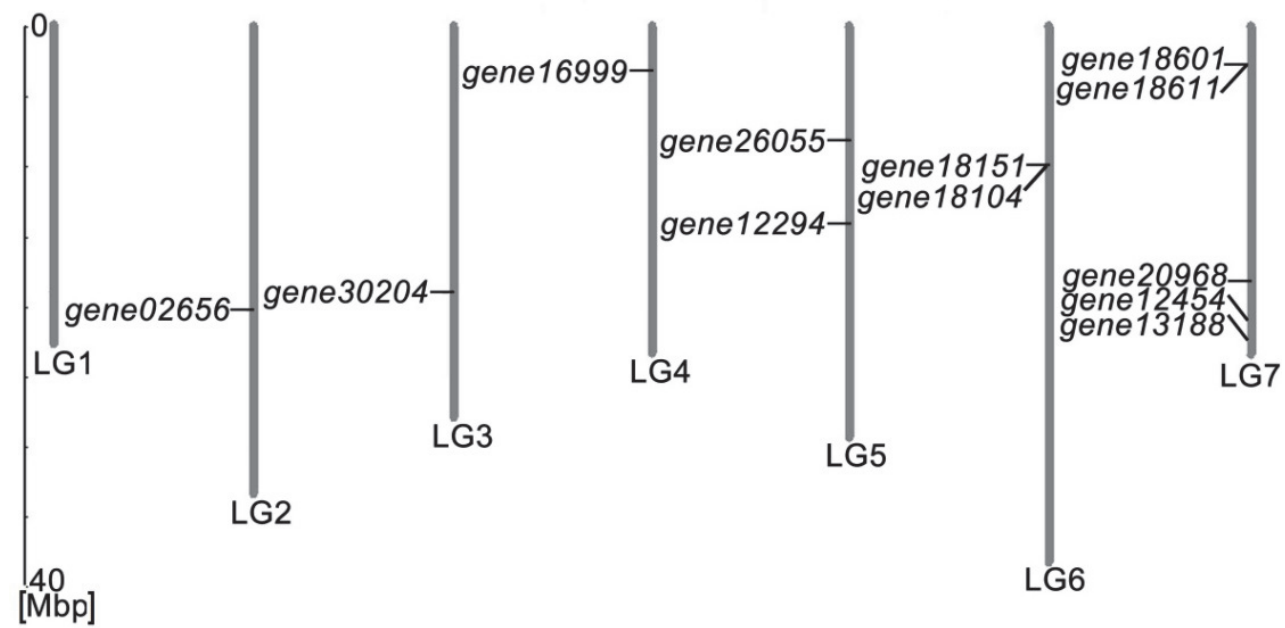

Fig. 1. Chromosomal locations of circadian clock genes on the seven chromosomes of Fragaria vesca. The scale on the left is in megabases.

Table 2. Hormone- or stress-related and tissue- or organ-specific cis-elements in the promoter regions of circadian clock genes in F. vesca. ${ }^{a}$ - The different cis-elements are represented by numbers: 1 - fungal elicitor, 2 - elicitor, 3 - anaerobic induction, 4 - irradiance, 5 - heat stress, 6 - low temperature, 7 - defense and stress, 8 - wounding, 9 - ABA, 10 - SA, 11 - MeJA, 12 - ethylene, 13 - GA, 14 - auxin, 15 - meristem, 16 - endosperm, 17 - shoot, 18 - cell cycle, 19 - seed, 20 - metabolism, 21 - MYB binding site, 22 - circadian control. ${ }^{\mathrm{b}}$ - numbers in columns indicate the number of the cis-elements.

\begin{tabular}{|c|c|c|c|c|c|c|c|c|c|c|c|c|c|c|c|c|c|c|c|c|c|c|}
\hline & $1^{\mathrm{a}}$ & 2 & 3 & 4 & 5 & 6 & 7 & 8 & 9 & 10 & 11 & 12 & 13 & 14 & 15 & 16 & 17 & 18 & 19 & 20 & 21 & 22 \\
\hline$F v C C A 1$ & $2^{b}$ & & 2 & 10 & & & 2 & & 2 & 1 & & & & & 1 & 3 & & & & & & 1 \\
\hline$F v P R R 9$ & & & 3 & 37 & 4 & & 1 & & 8 & & 4 & 1 & & & 1 & 4 & & & & & 2 & 4 \\
\hline$F v P R R 7$ & & & 6 & 22 & & & 1 & & 2 & 1 & 6 & & 1 & 1 & 1 & 4 & & & & & 4 & \\
\hline$F v R V E 8$ & 1 & & 2 & 18 & 1 & & & 1 & 2 & 2 & 6 & 1 & 2 & 1 & & 9 & 1 & & & 1 & 1 & 1 \\
\hline$F v R E V 6$ & 1 & & 1 & 27 & & & 1 & & & & 4 & & & 1 & 2 & 2 & & & & 1 & 3 & 2 \\
\hline$F v P R R 5$ & & & & 31 & 1 & & 2 & & 5 & 1 & 4 & & 3 & 1 & & 3 & & 2 & & 1 & 1 & 2 \\
\hline FvTOC1 & 1 & & 2 & 16 & 1 & 2 & 1 & & & 1 & 2 & 1 & 1 & & 1 & 5 & & & & & & 3 \\
\hline$F v C H E$ & & & & 22 & 2 & 1 & & 1 & & & 2 & 3 & & 1 & & 1 & & & 1 & & 1 & 3 \\
\hline$F v L U X$ & 2 & 1 & 2 & 14 & 2 & & 4 & & 2 & & 6 & & & & 2 & 4 & & & & 1 & 1 & 3 \\
\hline$F v E L F 3$ & 2 & & 1 & 17 & & 1 & 1 & & 4 & 2 & 4 & & & 1 & & 1 & & & & 1 & & 3 \\
\hline$F v E L F 4$ & 2 & & & 17 & & 2 & & & 1 & 1 & 2 & & 3 & & 1 & & & & & 1 & & 2 \\
\hline$F v Z T L$ & 1 & & & 21 & 4 & & & & & 1 & 4 & & 1 & & 2 & 2 & & & & & 1 & 3 \\
\hline
\end{tabular}

To understand the potential regulatory mechanisms of circadian clock genes in $F$. vesca, the $1.5 \mathrm{~kb}$ sequences upstream of the translation start sites of the strawberry circadian clock genes were analysed using PlantCARE. A set of cis-regulatory elements involved in abiotic and biotic stress responses, phytohormone responses, and plant growth and development were identified (Table 2).
For instance, fungal elicitor responsive elements were found in eight circadian clock genes. All strawberry primary circadian clock genes had light-responsive elements. Heat-stress- and low-temperature-responsive elements were identified in 7 and 4 circadian clock genes, respectively. In addition, the promoter regions of 8 circadian clock genes contained defence and stress- 
responsive elements. Among phytohormone-related regulatory elements, elements responsive to abscisic acid (ABA), salicylic acid (SA), and methyl jasmonate were identified in the promoter regions of 8,8 , and 11 strawberry circadian clock genes, respectively. The elements responsive to ethylene, gibberellin (GA), and auxin were observed in 4, 6, and 6 strawberry circadian clock genes, respectively (Table 2). Many hormoneresponsive elements were identified in the promoter regions of strawberry circadian clock genes, suggesting that phytohormones might have a high degree of crosstalk with circadian clock genes. Additionally, many circadian clock genes contained meristem-, endosperm- and seedresponsive elements, indicating that strawberry circadian clock genes might have a role in regulating strawberry growth and development. Notably, the elements responsive to circadian-control were found in 11 strawberry circadian clock genes (Table 2 ).

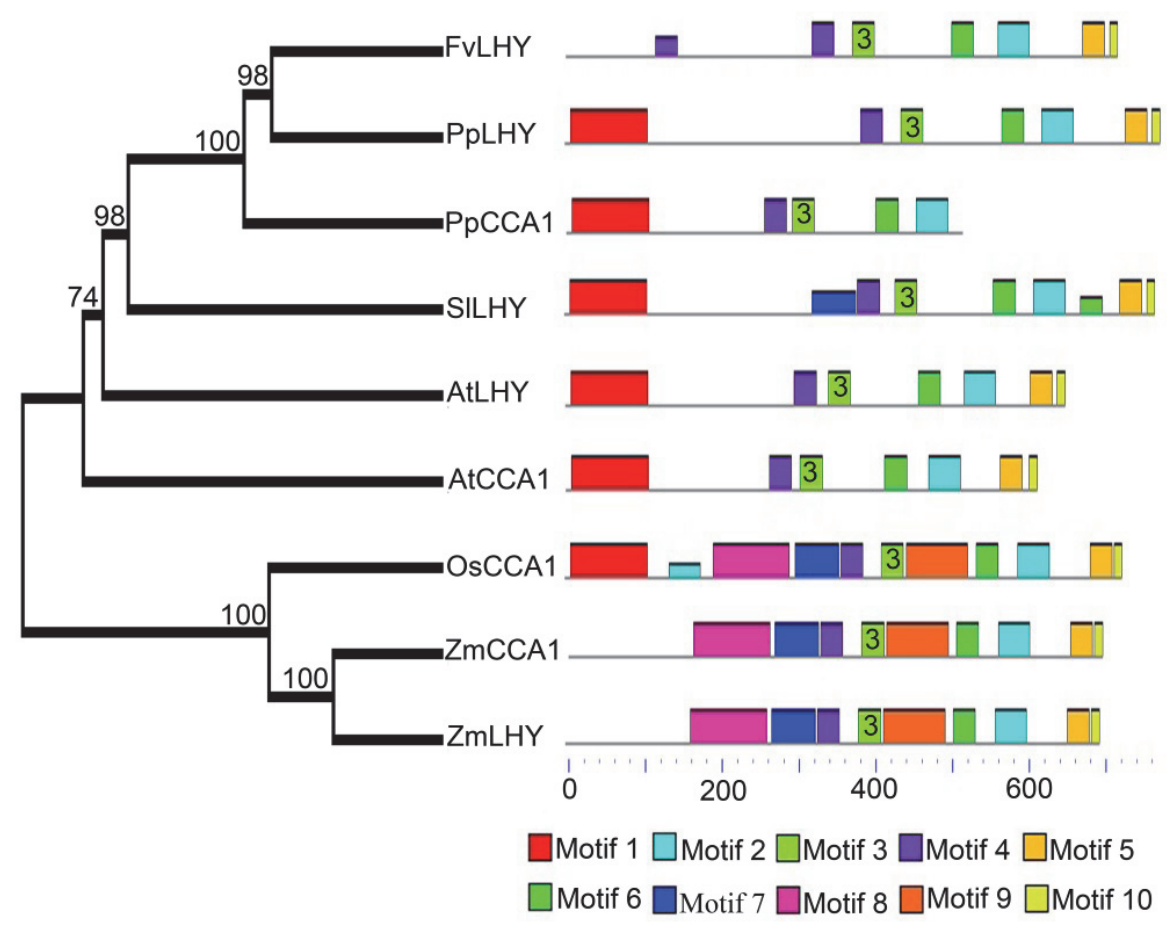

Fig. 2. Phylogenetic and motif analyses of LHY and CCA1 proteins. A phylogenetic tree was constructed using the full-length protein sequences of 9 LHY and CCA1 proteins retrieved from Arabidopsis thaliana (At), Prunus persica (Pp), Solanum lycopersicum $(S l)$, Zea mays $(\mathrm{Zm})$, Oryza sativa $(O s)$, and Fragaria vesca $(F v)$ databases. The protein motifs of the strawberry LHY and CCA1 proteins are shown below the graph and denoted by rectangles with different colours. To distinguish motif 3 and motif 6 , motif 3 is indicated by the number 3 . The sequences of these motifs are shown in Fig. 3 Suppl.

To test whether the expression of strawberry circadian clock genes exhibited circadian rhythms, we investigated their expression using real-time qPCR. The expression profiles of seven strawberry circadian clock genes displayed circadian rhythms (Fig. 4). For example, the mRNA abundance of FvLHY and FvRVE8 was high during the morning, declined gradually during the day, and was lowest during the afternoon and early night. The expression of FvPRR9, FvPRR7, and FvPRR5 peaked during the afternoon. Two evening-phased components ( $F v T O C 1$ and $F v L U X$ ) were expressed highly during the first half of the night (Fig. 4). On the other hand, the expression profiles of FvRVE6, FvCHE, FvELF3, and FvZTL showed no obvious circadian rhythms in strawberry (Fig. 4).

Plant circadian clock genes play important roles in regulating low-temperature stress response (Eriksson and
Webb 2011). To investigate the potential roles of strawberry circadian clock genes during plant responses to low-temperature stress, we analysed the expression profiles of 11 strawberry circadian clock genes in the leaves of $F$. vesca plantlets exposed to low-temperature stress (Fig. 5). In general, low-temperature stress led to the upregulation of morning-phase and day-phase components. For instance, the expression of $F v L H Y$ and $F v R V E 8$ was induced by cold stress from ZT4 to ZT16, while FvPRR9, FvPRR7, FvPRR5, and FvRVE6 were upregulated by cold treatment for $24 \mathrm{~h}$ (Fig. 5). On the other hand, low-temperature stress inhibited the induction of FvTOC1, FvLUX,FvELF3, and FvZTL during the night (Fig. 5). These data suggest different circadian clock genes might have different functions in response to low-temperature stress. 


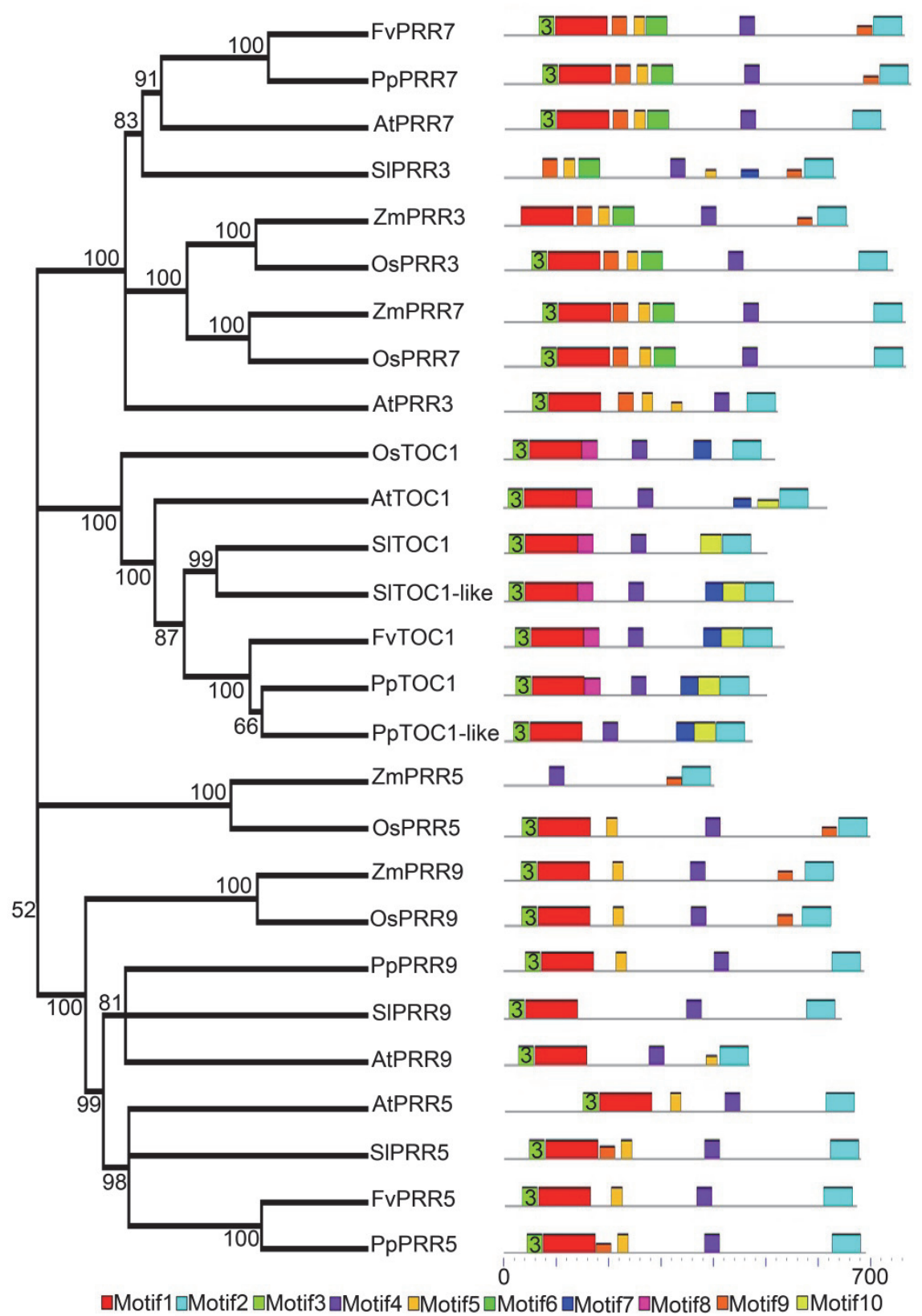

Fig. 3. Phylogenetic and motif analyses of PRR proteins. An unrooted tree was constructed using full-length PRR protein sequences from six different species (for their names and abbreviations see Fig. 2). The protein motifs (numbered 1 - 10) are shown below the graph, and the sequences of these motifs are shown in Fig. 4 Suppl.

\section{Discussion}

Circadian rhythms are driven and regulated by a number of circadian clock components. These circadian clock components function at different times of the day to regulate many biological processes (Bendix et al. 2015). Although the overall architecture of the circadian clock is generally conserved, the key genes involved often vary among organisms (Linde et al. 2017). In the present work, a total of 12 primary circadian clock genes were identified in the $F$. vesca genome (accession Hawaii-4). It is noteworthy that the homologues of AtCCA1, AtRVE4, $A t P R R 3$, and $A t B O A$ were absent in the $F$. vesca genome. CCA1 and LHY play important roles in regulating the morning-phase circadian rhythm in Arabidopsis (Mizoguchi et al. 2002, Hsu and Harmer 2014). Most plant species have only one of CCAI and $L H Y$, usually LHY (Calixto et al. 2015), and strawberry contains only $F v L H Y$. The expression data also demonstrated that $F v L H Y$ was expressed highly at the beginning of the day (Fig. 4). 


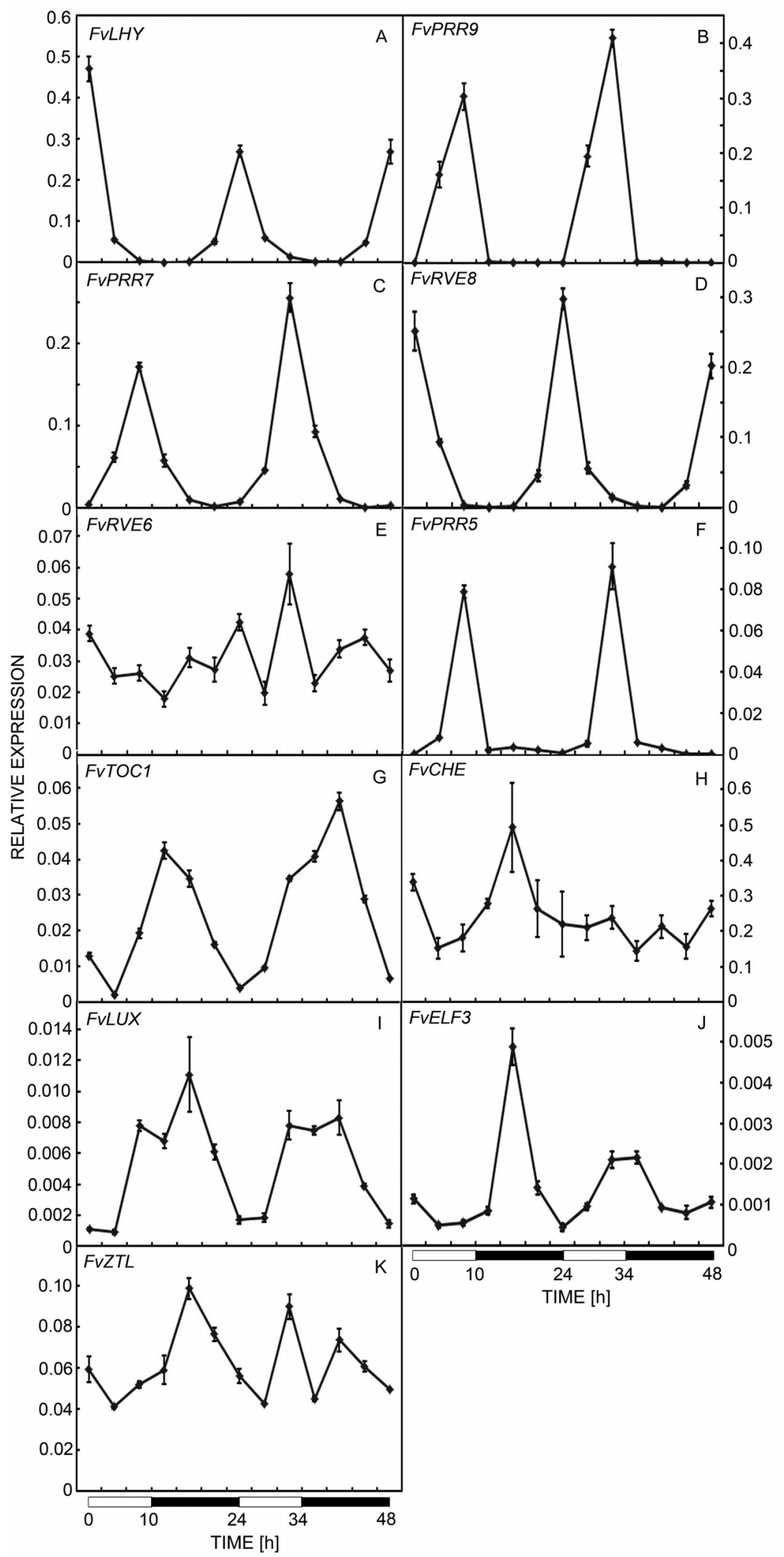

Fig. 4. Expression patterns of circadian clock genes in Fragaria vesca. Eleven circadian clock gene expression profiles were measured by real-time qPCR. Means \pm SDs were obtained from three biological replicates and three technical replicates. Leaves were collected from the beginning of the light period (ZT0) at 4-h intervals until ZT48. White and black bars on the x-axis indicate day and night periods, respectively. 


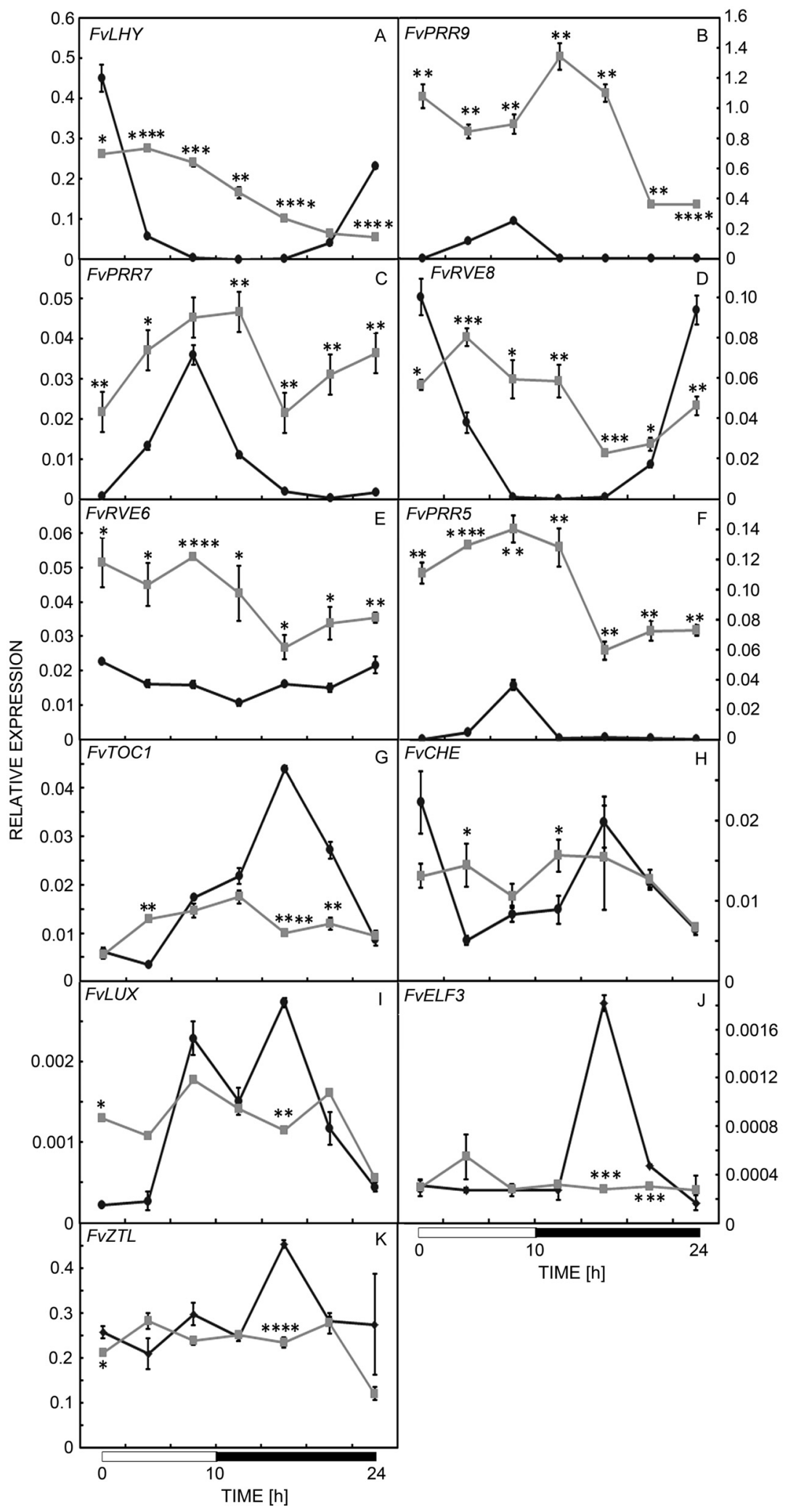

Fig. 5. Expression patterns of circadian clock genes in Fragaria vesca in response to low-temperature stress generated by real-time qPCR. Means \pm SDs from three biological replicates and three technical replicates. $*, * *, * * *$, and $* * * *$ indicate significant differences between the treated (grey lines) and control plants (black lines) at $P \leq 0.05,0.01,0.001$, and 0.0001 , respectively. Leaves were collected from ZT0 at 4-h intervals until ZT24. White and black bars on the x-axis indicate day and night period, respectively. 
These results suggest that $F v L H Y$ is necessary for the maintenance of morning-phase circadian rhythm in strawberry. The TOC1 genes (also known as $P R R 1$ ), together with PRR9, PRR7, PRR5, and PRR3, belong to the PRR gene family (Matsushika et al. 2000). The expression of these genes is regulated by the circadian clock, although their transcripts peak at different times of the day: PRR9 at dawn, PRR7 in the morning, PRR5 around noon, and TOC1 and PRR3 in the evening (Matsushika et al. 2000, Nakamichi et al. 2010). In $F$. vesca, the expression of FvPRR9, FvPRR7 and FvPRR5 was greatest around ZT8 (noon). These data indicate that the regulation of afternoon-phased circadian rhythms might be mainly regulated by these three PRR genes.

PRR5, TOC1, CHE, LUX, BOA, ELF3, ELF4, and $Z T L$ are involved in regulating the circadian clock during the evening in Arabidopsis (Hsu and Harmer 2014). However, we failed to identify a homologue of AtBOA in the diploid $F$. vescay genome. The expressions of $A t C H E$ (Pruneda-Paz et al. 2009) and AtELF3 (Lu et al. 2012) are clock-regulated, but their strawberry homologues did not show any obvious circadian rhythms. Only two evening-phase genes (FvTOC1 and FvLUX) showed periodic expression patterns. These results suggest that FvTOC1 and FvLUX might play pivotal roles in regulation of the circadian clock during the evening.

The plant circadian clock system influences the lowtemperature stress response (Eriksson and Webb 2011, Hsu and Harmer 2014). The best-characterized lowtemperature stress response pathway is the C-repeat (CRT)-binding factor/dehydration-responsive element (DRE) binding factor (CBF/DREB)-mediated transcriptional regulatory pathway (Chinnusamy et al. 2007, Shi et al. 2015). Three members of the $C B F / D R E B 1$ family, $C B F 1, C B F 2$, and $C B F 3$ (or $D R E B 1 b, D R E B 1 c$, and DREBIa, respectively) are rapidly induced by low temperatures (Fowler and Thomashow 2002, Medina et al. 2011). In addition, the induction of these $C B F$ genes was directly activated by

\section{References}

Alabadi, D., Yanovsky, M.J., Mas, P., Harmer, S.L., Kay, S.A.: Critical role for $C C A 1$ and $L H Y$ in maintaining circadian rhythmicity in Arabidopsis. - Curr. Biol. 12: 757-761, 2002.

Bailey, T.L., Boden, M., Buske, F.A., Frith, M., Grant, C.E., Clementi, L., Ren, J., Li, W.W., Noble, W.S.: MEME SUITE: tools for motif discovery and searching. - Nucl. Acids Res. 37: W202-W208, 2009.

Bendix, C., Marshall, C.M., Harmon, F.G.: Circadian clock genes universally control key agricultural traits. - Mol. Plant 8: 1135-1152, 2015.

Calixto, C.P., Waugh, R., Brown, J.W.: Evolutionary relationships among barley and Arabidopsis core circadian clock and clock-associated genes. - J. mol. Evol. 80: 108119, 2015.

Campoli, C., Shtaya, M., Davis, S.J., Von Korff, M.: Expression conservation within the circadian clock of a monocot: natural variation at barley $P p d-H 1$ affects circadian expression of flowering time genes, but not clock orthologs.
CCA1 and LHY in Arabidopsis (Dong et al. 2011) and might be negatively regulated by $P R R 5, P R R 7$, and $P R R 9$ (Nakamichi et al. 2009). Our current data show that the expression of morning-phase components (FvLHY) and day-phase components ( FvPRR5, FvPRR7, and FvPRR9) were induced by cold treatment, whereas evening-phase components were slightly downregulated by lowtemperature stress. These results suggest that morningphase and day-phase circadian clock genes ( $F v L H Y$, $F v P R R 5, F v P R R 7$, and $F v P R R 9$ ) might play pivotal roles in coordinating circadian clock regulation under lowtemperature stress in strawberry. As the expression of $C B F$ genes was gated by morning-phase components (CCA1/LHY) in Arabidopsis and the expression of $F v L H Y$ was induced by cold treatment, we speculate that the induction of $F v C B F$ genes might also be regulated by $F v L H Y$. However, the molecular function of the circadian clock genes in regulating low-temperature response is still poorly understood in strawberry. Further detailed work on the regulation and function of these circadian clock genes in strawberry will greatly increase our knowledge of how strawberry circadian genes are involved in the regulation of low-temperature stress.

In conclusion, we performed a broad study of circadian clock genes in $F$. vesca by investigating their evolutionary relationships, protein motif distributions, gene structure, and cis-regulatory elements. Among these genes, we found that seven strawberry circadian clock genes (FvLHY, FvPRR9, FvPRR7, FvRVE8, FvPRR5, $F v T O C 1$, and $F v L U X)$ had typical circadian expression patterns, which suggest that these genes might play central roles in regulating circadian rhythms in strawberry. In addition, we examined the expression profiles of these circadian clock genes under low-temperature stress, which provided additional insights into their functions. Taken together, our research lays the foundation for future work to reveal the functions of circadian clock genes in strawberry.

- BMC Plant Biol. 12: 97, 2012.

Chinnusamy, V., Zhu, J., Zhu, J. K.: Cold stress regulation of gene expression in plants. - Trends Plant Sci. 12: 444-451, 2007.

Dong, G.G., Golden, S.S.: How a cyanobacterium tells time. Curr. Opin. Microbiol. 11: 541-546, 2008.

Dong, M.A., Farre, E.M., Thomashow, M.F.: Circadian clockassociated 1 and late elongated hypocotyl regulate expression of the C-repeat binding factor (CBF) pathway in Arabidopsis. - Proc. nat. Acad. Sci. USA 108: 7241-7246, 2011.

Eriksson, M.E., Webb, A.A.: Plant cell responses to cold are all about timing. - Curr. Opin. Plant Biol. 14: 731-737, 2011.

Fowler, S., Thomashow, M. F.: Arabidopsis transcriptome profiling indicates that multiple regulatory pathways are activated during cold acclimation in addition to the CBF cold response pathway. - Plant Cell 14: 1675-1690, 2002.

Fukushima, A., Kusano, M., Nakamichi, N., Kobayashi, M., 
Hayashi, N., Sakakibara, H., Mizuno, T., Saito, K.: Impact of clock-associated Arabidopsis pseudo-response regulators in metabolic coordination. - Proc. nat. Acad. Sci. USA 106: 7251-7256, 2009.

Greenham, K., McClung, C.R.: Integrating circadian dynamics with physiological processes in plants. - Nat. Rev. Genet. 16 598-610, 2015.

Gu, T., Ren, S., Wang, Y., Han, Y., Li, Y.: Characterization of DNA methyltransferase and demethylase genes in Fragaria vesca. - Mol. Genet. Genomics 291: 1333-1345, 2016.

Herrero, E., Kolmos, E., Bujdoso, N., Yuan, Y., Wang, M., Berns, M. C., Uhlworm, H., Coupland, G., Saini, R., Jaskolski, M., Webb, A., Goncalves, J., Davis, S. J.: EARLY FLOWERING4 recruitment of EARLY FLOWERING3 in the nucleus sustains the Arabidopsis circadian clock. - Plant Cell 24: 428-443, 2012.

Hsu, P.Y., Harmer, S.L.: Wheels within wheels: the plant circadian system. - Trends Plant Sci. 19: 240-249, 2014.

Hu, B., Jin, J., Guo, A.Y., Zhang, H., Luo, J., Gao, G.: GSDS 2.0: an upgraded gene feature visualization server. Bioinformatics 31: 1296-1297, 2015.

Ibanez, C., Ramos, A., Acebo, P., Contreras, A., Casado, R., Allona, I., Aragoncillo, C.: Overall alteration of circadian clock gene expression in the chestnut cold response. - PLoS ONE 3: e3567, 2008.

Jung, S., Ficklin, S.P., Lee, T., Cheng, C.H., Blenda, A., Zheng, P., Yu, J., Bombarely, A., Cho, I., Ru, S., Evans, K., Peace, C., Abbott, A.G., Mueller, L.A., Olmstead, M.A., Main, D.: The genome database for Rosaceae (GDR): year 10 update. - Nucl. Acids Res. 42: D1237-D1244, 2014.

Lamesch, P., Berardini, T.Z., Li, D., Swarbreck, D., Wilks, C., Sasidharan, R., Muller, R., Dreher, K., Alexander, D.L., Garcia-Hernandez, M., Karthikeyan, A.S., Lee, C.H., Nelson, W.D., Ploetz, L., Singh, S., Wensel, A., Huala, E.: The Arabidopsis Information Resource (TAIR): improved gene annotation and new tools. - Nucl. Acids Res. 40: D1202-D1210, 2012.

Lescot, M., Dehais, P., Thijs, G., Marchal, K., Moreau, Y., Van de Peer, Y., Rouze, P., Rombauts, S.: PlantCARE, a database of plant cis-acting regulatory elements and a portal to tools for in silico analysis of promoter sequences. - Nucl. Acids Res. 30: 325-327, 2002.

Linde, A.-M., Eklund, D.M., Kubota, A., Pederson, E.R.A., Holm, K., Gyllenstrand, N., Nishihama, R., Cronberg, N., Muranaka, T., Oyama, T., Kohchi, T., Lagercrantz, U.: Early evolution of the land plant circadian clock. - New Phytol. 216: 576-590, , 2017.

Lou, P., Wu, J., Cheng, F., Cressman, L. G., Wang, X., McClung, C. R.: Preferential retention of circadian clock genes during diploidization following whole genome triplication in Brassica rapa. - Plant Cell 24: 2415-2426, 2012.

Lu, S.X., Webb, C. J., Knowles, S.M., Kim, S.H., Wang, Z., Tobin, E.M.: CCA1 and ELF3 interact in the control of hypocotyl length and flowering time in Arabidopsis. - Plant Physiol. 158: 1079-1088, 2012.

Maibam, P., Nawkar, G.M., Park, J.H., Sahi, V.P., Lee, S.Y., Kang, C.H.: The influence of light quality, circadian rhythm, and photoperiod on the CBF-mediated freezing tolerance. Int. J. mol. Sci. 14: 11527-11543, 2013.

Marcolino-Gomes, J., Rodrigues, F.A., Fuganti-Pagliarini, R., Bendix, C., Nakayama, T.J., Celaya, B., Molinari, H.B.C., De Oliveira, M.C.N., Harmon, F.G., Nepomuceno, A.: Diurnal oscillations of soybean circadian clock and drought responsive genes. - PLoS ONE 9: e86402, 2014.
Mas, P., Kim, W.Y., Somers, D.E., Kay, S.A.: Targeted degradation of TOC1 by ZTL modulates circadian function in Arabidopsis thaliana. - Nature 426: 567-570, 2003.

Matsushika, A., Makino, S., Kojima, M., Mizuno, T.: Circadian waves of expression of the APRR1/TOC1 family of pseudoresponse regulators in Arabidopsis thaliana: insight into the plant circadian clock. - Plant Cell Physiol. 41: 1002-1012, 2000.

Medina, J., Catala, R., Salinas, J.: The CBFs: three arabidopsis transcription factors to cold acclimate. - Plant Sci. 180: 3-11, 2011.

Mizoguchi, T., Wheatley, K., Hanzawa, Y., Wright, L., Mizoguchi, M., Song, H.R., Carre, I.A., Coupland, G.: LHY and $C C A 1$ are partially redundant genes required to maintain circadian rhythms in Arabidopsis. - Develop. Cell 2: 629-641, 2002.

Mouhu, K., Hytonen, T., Folta, K., Rantanen, M., Paulin, L., Auvinen, P., Elomaa, P.: Identification of flowering genes in strawberry, a perennial SD plant. - BMC Plant Biol. 9: $122,2009$.

Nakamichi, N., Kiba, T., Henriques, R., Mizuno, T., Chua, N. H., Sakakibara, H.: PSEUDO-RESPONSE REGULATORS 9 , 7 , and 5 are transcriptional repressors in the Arabidopsis circadian clock. - Plant Cell 22: 594-605, 2010.

Nakamichi, N., Kusano, M., Fukushima, A., Kita, M., Ito, S., Yamashino, T., Saito, K., Sakakibara, H., Mizuno, T.: Transcript profiling of an Arabidopsis PSEUDO RESPONSE REGULATOR arrhythmic triple mutant reveals a role for the circadian clock in cold stress response. - Plant Cell Physiol. 50: 447-462, 2009.

Nusinow, D.A., Helfer, A., Hamilton, E.E., King, J.J., Imaizumi, T., Schultz, T.F., Farre, E.M., Kay, S.A.: The ELF4-ELF3LUX complex links the circadian clock to diurnal control of hypocotyl growth. - Nature 475: 398-402, 2011.

Pruneda-Paz, J.L., Breton, G., Para, A., Kay, S.A.: A functional genomics approach reveals $\mathrm{CHE}$ as a component of the Arabidopsis circadian clock. - Science 323: 1481-1485, 2009.

Rawat, R., Takahashi, N., Hsu, P.Y., Jones, M.A., Schwartz, J., Salemi, M.R., Phinney, B.S., Harmer, S.L.: REVEILLE8 and PSEUDO-REPONSE REGULATOR5 form a negative feedback loop within the Arabidopsis circadian clock. PLoS Genet. 7: e1001350, 2011.

Schmittgen, T.D., Livak, K.J.: Analyzing real-time PCR data by the comparative CT method. - Natur. Protocols 3: 11011108,2008

Shi, Y., Ding, Y., Yang, S.: Cold signal transduction and its interplay with phytohormones during cold acclimation. Plant Cell Physiol. 56: 7-15, 2015.

Shulaev, V., Sargent, D.J., Crowhurst, R.N., Mockler, T.C., Folkerts, O., Delcher, A.L., Jaiswal, P., Mockaitis, K., Liston, A., Mane, S.P., Burns, P., Davis, T.M., Slovin, J.P., Bassil, N., Hellens, R.P., Evans, C., Harkins, T., Kodira, C., Desany, B., Crasta, O.R., Jensen, R.V., Allan, A.C., Michael, T.P., Setubal, J.C., Celton, J.M., Rees, D.J., Williams, K.P., Holt, S.H., Ruiz Rojas, J.J., Chatterjee, M., Liu, B., Silva, H., Meisel, L., Adato, A., Filichkin, S.A., Troggio, M., Viola, R., Ashman, T.L., Wang, H., Dharmawardhana, P., Elser, J., Raja, R., Priest, H.D., Bryant, D.W., Jr., Fox, S.E., Givan, S.A., Wilhelm, L.J., Naithani, S., Christoffels, A., Salama, D.Y., Carter, J., Lopez Girona, E., Zdepski, A., Wang, W., Kerstetter, R.A., Schwab, W., Korban, S.S., Davik, J., Monfort, A., DenoyesRothan, B., Arus, P., Mittler, R., Flinn, B., Aharoni, A., Bennetzen, J.L., Salzberg, S.L., Dickerman, A.W., Velasco, 
R., Borodovsky, M., Veilleux, R.E., Folta, K.M.: The genome of woodland strawberry (Fragaria vesca). - Nat. Genet. 43: 109-116, 2011.

Tamura, K., Peterson, D., Peterson, N., Stecher, G., Nei, M., Kumar, S.: MEGA5: molecular evolutionary genetics analysis using maximum likelihood, evolutionary distance, and maximum parsimony methods. - Mol. Biol. Evol. 28: 2731-2739, 2011.

Wang, Y.Q., Ni, X., Yan, J., Yang, L.: Modeling transcriptional co-regulation of mammalian circadian clock. - Math. Biosci.
Eng. 14: 1447-1462, 2017.

Wei, W., Hu, Y., Cui, M.Y., Han, Y.T., Gao, K., Feng, J.Y.: Identification and transcript analysis of the TCP transcription factors in the diploid woodland strawberry Fragaria vesca. - Front. Plant Sci. 7: 1937, 2016.

Yang, Y., Peng, Q., Chen, G.X., Li, X.H., Wu, C.Y.: OsELF3 is involved in circadian clock regulation for promoting flowering under long-day conditions in rice. - Mol. Plant 6: 202-215, 2013. 